\title{
Semi-Mechanistic Modeling of Florfenicol Time-Kill Curves and in silico Dose Fractionation for Calf Respiratory Pathogens
}

\author{
Ludovic Pelligand ${ }^{1 *}$, Peter Lees ${ }^{1}$, Pritam Kaur Sidhu ${ }^{2}$ and Pierre-Louis Toutain ${ }^{1,3}$ \\ ${ }^{1}$ Royal Veterinary College, Department of Comparative Biomedical Sciences, Hawkshead Campus, Hatfield, \\ United Kingdom, ${ }^{2}$ Institute of Computational Comparative Medicine, College of Veterinary Medicine, Kansas State \\ University, Manhattan, KS, United States, ${ }^{3}$ École Nationale Vétérinaire de Toulouse, Toulouse, France
}

OPEN ACCESS

Edited by:

Ghassan M. Matar, American University of Beirut,

Lebanon

Reviewed by:

Lena Friberg,

Uppsala University, Sweden

Cengiz Gokbulut,

Balıkesir University, Turkey

*Correspondence:

Ludovic Pelligana

Ipelligand@rvc.ac.uk

Specialty section:

This article was submitted to

Antimicrobials, Resistance

and Chemotherapy,

a section of the journal

Frontiers in Microbiology

Received: 11 April 2019

Accepted: 17 May 2019

Published: 11 June 2019

Citation:

Pelligand L, Lees P, Sidhu PK and

Toutain P-L (2019) Semi-Mechanistic

Modeling of Florfenicol Time-Kill

Curves and in silico Dose

Fractionation for Calf Respiratory

Pathogens. Front. Microbiol. 10:1237.

doi: 10.3389/fmicb.2019.01237
An important application of time-kill curve (TKC) assays is determination of the nature of the best PK/PD index ( $f A U C / M I C$ or $f \mathrm{~T} \%>\mathrm{MIC}$ ) and its target value for predicting clinical efficacy in vivo. VetCAST (the veterinary subcommittee of EUCAST) herein presents semi-mechanistic TKC modeling for florfenicol, a long acting (96h) veterinary antimicrobial drug licensed against calf pneumonia organisms (Pasteurella multocida and Mannheimia haemolytica) to support justification of its PK/PD breakpoint and clinical breakpoint. Individual TKC assays were performed with 6 field strains of each pathogen (initial inoculum $10^{7} \mathrm{CFU} / \mathrm{mL}$ with sampling at times at $0,1,2,4,8$, and $24 \mathrm{~h}$ ). Semi-mechanistic modeling (Phoenix NLME) allowed precise estimation of bacteria growth system ( $K_{G R O W T H}$, natural growth rate; $K_{D E A T H}$, death rate; $B_{M A X}$, maximum possible culture size) and florfenicol pharmacodynamic parameters ( $E_{M A X}$, efficacy additive to $\mathrm{K}_{\mathrm{DEATH}}$; $\mathrm{EC}_{50}$, potency; Gamma, sensitivity). PK/PD simulations (using the present TKC model and parameters of a florfenicol population pharmacokinetic model) predicted the time-course of bacterial counts under different exposures. Of two licensed dosage regimens, $40 \mathrm{mg} / \mathrm{kg}$ administered once was predicted to be superior to $20 \mathrm{mg} / \mathrm{kg}$ administered at $48 \mathrm{~h}$ intervals. Furthermore, we performed in silico dose fractionation with doses $0-80 \mathrm{mg} / \mathrm{kg}$ administered in 1,2 or 4 administrations over $96 \mathrm{~h}$ and for MICs of $0.5,1,2,4 \mathrm{mg} / \mathrm{L}$ with 2 inoculum sizes $10^{5}$ and $10^{7} \mathrm{CFU} / \mathrm{mL}$. Regression analysis ( $I_{\max }$ model) demonstrated that i) fAUC/MIC outperformed $f \mathrm{~T} \%>$ MIC as PK/PD index and ii) maximum efficacy ( $\mathrm{IC}_{90 \%}$ ) was obtained when the average free plasma concentration over $96 \mathrm{~h}$ was equal to 1.2 to 1.4 times the MIC of Pasteurella multocida and Mannheimia haemolytica, respectively.

\footnotetext{
Keywords: PK/PD, modeling and simulation, time-kill assay, antimicrobial susceptibility testing, Pasteurella multocida, Mannheimia haemolytica, VetCAST, bovine respiratory disease
}

\section{INTRODUCTION}

The aim of time-kill in vitro assays is to investigate the pharmacodynamics (PD) of antimicrobial drugs (AMD) by determining the rate of bacterial kill relative to drug concentration. Quantitative analysis of time-kill curve (TKC) data is more informative of the drug-bacteria relationship than snapshot indices, such as minimum inhibitory concentration (MIC). MIC 
indicates only the net effect of a single AMD concentration on bacterial growth over a $24 \mathrm{~h}$ incubation period, while TKC establishes the rate of killing over a range of concentrations. Based on TKC data AMDs can be classified as time- or concentration-dependent in killing action (Toutain et al., 2017).

An important application of TKC data is determination of the best $\mathrm{PK} / \mathrm{PD}$ index ( $f$ AUC/MIC or $f \mathrm{~T}>\mathrm{MIC}$ ) for predicting clinical efficacy in vivo, where $f A U C$ is area under plasma concentration-time curve and $f \mathrm{~T}$ is the time the drug concentration exceeds MIC, for free drug concentrations. This has historically been established by correlating the reduction in bacterial count at $24 \mathrm{~h}$ from an initial inoculum count (Lees et al., 2015). Plots of $\log 10$ colony forming units (CFU)/mL at $24 \mathrm{~h}$ versus each of the two PK/PD indices allowed selection of the $\mathrm{PK} / \mathrm{PD}$ index which best fits the sigmoidal $\mathrm{E}_{\mathrm{MAX}}$ model (Lees et al., 2015). This approach was based on the net reduction of bacterial count with each concentration exposure, but did not utilize the time course (i.e., the shape) of individual kill curves. For human medicine, several advanced PK/PD models of TKC have incorporated the shape of the curve with time (Nielsen et al., 2007; Nielsen and Friberg, 2013, model D, Figure 5). This more advanced modeling enables estimation of the three pharmacodynamic (PD) parameters of AMD action, namely potency, efficacy and sensitivity. This approach therefore allows characterization the whole concentration-effect relationship.

In the present investigation, using historical TKC data (Sidhu et al., 2014), PD parameters of florfenicol against the calf pneumonia organisms, Pasteurella multocida (P. multocida) and Mannheimia haemolytica ( $M$. haemolytica), have been established using the semi-mechanistic model proposed by Nielsen and Friberg (2013). The objective was to then conduct an in silico dose fractionation trial to determine the $\mathrm{PK} / \mathrm{PD}$ index for florfenicol and these pathogens, best correlating with bacterial kill. Dose fractionation studies are generally conducted in vivo using rodent infection models, whereas in this study semi-mechanistic PD florfenicol models were used as a surrogate of rodent models to predict microbiological effects in response to a range of florfenicol dosage regimens. The ultimate goal was to compute a $\mathrm{PK} / \mathrm{PD}$ breakpoint $\left(\mathrm{PK} / \mathrm{PD}_{\mathrm{BP}}\right)$ for the florfenicol clinical breakpoint (CBP) according to the procedures advocated by VetCAST (Toutain et al., 2017), where $\mathrm{CBP}$ is the MIC value used by microbiology laboratories to report the results of antimicrobial sensitivity testing (AST). $\mathrm{PK} / \mathrm{PD} \mathrm{CO}$ is defined as the highest possible MIC for which a given percentage of animals in the target population (say 90\%) achieve a pre-defined target value of the PK/PD index, the pharmacodynamic target (PDT) according to European Medicines Agency (EMA) terminology.

\section{MATERIALS AND METHODS}

\section{Test Pathogens and MIC Determination}

The test pathogens were $P$. multocida and M. haemolytica. MICs were determined in Mueller Hinton Broth (MHB) for six strains of each species, isolated from cases of calf pneumonia (Sidhu et al., 2014). Their origin and date of isolation are summarized in the Supplementary File S1. Average florfenicol MICs were determined using 5 overlapping 2-fold-dilution series and were 0.4 and $0.5 \mathrm{mg} / \mathrm{L}$ for $P$. multocida and M. haemolytica, respectively.

\section{Time-Kill Curves}

Six individual TK assays were performed for each pathogen. Initial inoculum count was in the range $5 \times 10^{6}$ to $7 \times 10^{7} \mathrm{CFU} / \mathrm{mL}$. Duration of incubation was $24 \mathrm{~h}$ with sampling at times of $0,1,2,4,8$, and $24 \mathrm{~h}$. Drug concentrations were expressed in the initial publication (Sidhu et al., 2014) as multiples of MIC ( 0 = growth control, $0.25,0.5,1,2$, and 4 times measured MIC). For data fitting, MICs were back calculated to $\mathrm{mg} / \mathrm{L}$. The lowest detectable count was $33 \mathrm{CFU} / \mathrm{mL}$; lower counts were set as below the quantification limit (BQL). All TKC data sets analyzed for this study are included in the manuscript and the supplementary File $\mathbf{S} 2$.

\section{Data Analysis}

Pharmacodynamic data analyses were conducted using Phoenix ${ }^{\circledR}$ WinNonlin $^{\circledR} \quad 8.0$ (Pharsight Corporation, St Louis, MO, United States). For each pathogen, the 6 TKC data sets were analyzed simultaneously using a non-linear mixed effect model (NLME). A semi-mechanistic structural model of bacterial growth, incorporating a compartment for growing drug-sensitive bacteria $(\mathrm{S})(\mathrm{CFU} / \mathrm{mL})$ and a compartment named persisters (P) $(\mathrm{CFU} / \mathrm{mL})$, corresponding to a pool of non-growing and insensitive-drug bacteria (phenotypic resistance) was adopted (Figure 1; Nielsen and Friberg, 2013).

Visual Inspection of TKC indicates an initial phase of slow growth. To capture the delay required to achieve a maximal steady-state growing rate, a mitigating function for $K_{G R O W T H}$ of the form was introduced (Equation 1):

$$
K_{G R O W T H}=K_{G R O W T H M A X} \times(1-\operatorname{EXP}(- \text { Alpha } \times \text { Time }))
$$

where Alpha (per h) = rate constant to describe a progressive increase of $K_{G R O W T H}$ over time; at time $0, K_{G R O W T H}=0$ then, $K_{\text {GROWTH }}$ increases progressively to reach $K_{\text {GROWTHMAX }}$ with a mean time equal to $1 / A l p h a$. The lag phase corresponds to the physiological adaptation of the bacteria to the culture condition (induction of specific messenger RNA and protein synthesis and low cell density accounting for initial dilution of the exoenzymes that make nutrients readily available).

Florfenicol action was introduced in the model as a concentration-dependent killing rate $K_{D R U G(t)}$ (per h) acting in parallel with $K_{D E A T H}$ but for the $\mathrm{S}$ pool only. It was modeled according to the classical Hill equation (Eq. 2).

$$
K_{D R U G(t)}=\frac{E_{M A X} \times C(t)^{\text {Gamma }}}{E C_{50}^{\text {Gamma }}+C(t)^{\text {Gamma }}}
$$

where $C(t)$ is the florfenicol concentration $(\mathrm{mg} / \mathrm{L})$ at time $t$ (the independent variable). $C(t)$ was the constant tested concentration when data were fitted to estimate the PD parameters but 


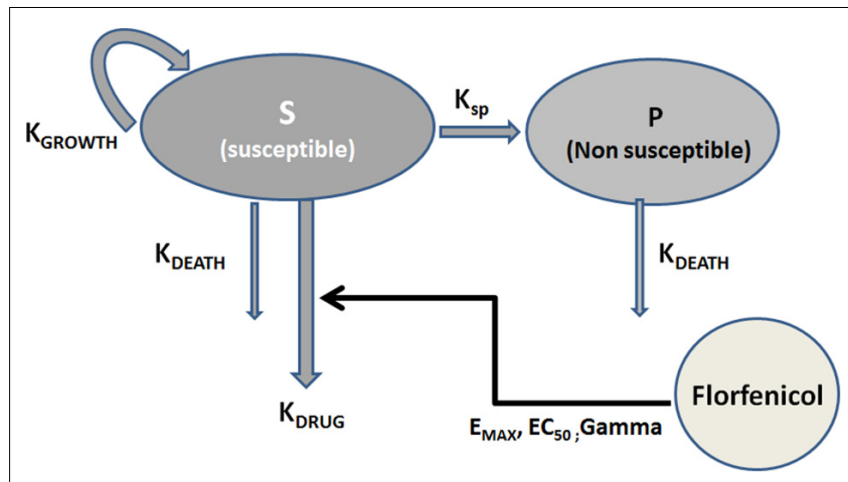

FIGURE 1 | Semi-mechanistic model for Time-Kill curve modeling, described in Nielsen et al. (2007) and Nielsen and Friberg (2013). $K_{\text {DEATH }}($ per $h$ ) is the natural death rate for both $S$ (susceptible) and $P$ (non-susceptible) pools; $K_{\text {GROWTH }}($ per $h)$ is the growth rate of $S$; $B_{M A X}(C F U / m L)$ is the maximum possible size of the culture $(S+P) . S+P$ constitutes the total bacterial count. For the starting inoculum, all bacteria were assumed to be in the growing, drug-sensitive stage. As the total bacterial content in the system increases, bacteria are progressively transferred into the $P$ resting pool. This transfer was modeled by an irreversible rate constant $\left(K_{S P}\right)$ between $S$ and $P . K_{S P}$ was parametrized in terms of $B_{M A X}, K_{G R O W T H}$ and $K_{D E A T H}$ (natural death rate) as described in Nielsen et al. (2007) and Nielsen and Friberg (2013). EMAX (1/h) is a maximal killing rate of bacteria for the susceptible pool (additional to natural death rate), $E C_{50}$ is the florfenicol in vitro concentration $(\mathrm{mg} / \mathrm{L})$ for $E_{M A X} / 2$ and Gamma (a scalar), the Hill coefficient; $E_{M A X}, E C_{50}$ and Gamma are the three PD parameters allowing quantification of florfenicol efficacy, potency and sensitivity, respectively.

$C(t)$ was obtained by solving the population PK model, when this equation was used for simulations (vide infra). $E_{M A X}$ $(1 / \mathrm{h})$ is the maximal killing rate for the susceptible pool (additionally to natural death rate), $E C_{50}$ is the florfenicol in vitro concentration (mg/L) for $\mathrm{E}_{\mathrm{MAX}} / 2$ and Gamma (a scalar), the Hill coefficient; $E_{M A X}, E C_{50}$ and Gamma are the three PD parameters providing quantitative indices of florfenicol efficacy, potency and sensitivity, respectively.

There were substantial differences in $B_{M A X}$ across the six field strains of each pathogen. Therefore, a random component was introduced in the structural model to account for the inter-strain variability. Six individual $B_{M A X}$ values were obtained, using an exponential model of the form (Eq. 3):

$$
\theta_{1 i}=\theta_{1} \times \operatorname{Exp}\left(\eta_{1 i}\right)
$$

where $\theta_{1}$ is the typical population value of $B_{M A X}, \theta_{1 i}$ the value of $B_{M A X}$ for the $i^{\text {th }}$ TKC assay, and $\eta_{1 i}$ (eta) the deviation associated with the $i^{\text {th }}$ strain from the corresponding population value. This exponential model assumes a log-normal distribution of $B_{M A X}$. The between-strain variability of $B_{M A X}$ was reported as coefficient of variation in the original scale, with an equation converting estimated variance terms to a coefficient of variation (CV\%) (Eq. 4).

$$
C V(\%)=100 \times \sqrt{\exp \left(\omega^{2}\right)-1}
$$

The residual variability was modeled with an exponential error model of the form (Eq. 5):

$$
Y_{i j}=\widehat{Y}_{i j} \times \operatorname{EXP}\left(\varepsilon_{i j}\right)
$$

where $\widehat{Y}_{\mathrm{ij}}$ is the $j^{\text {th }}$ response $(\mathrm{CFU} / \mathrm{mL})$ measured in the $i^{\text {th }}$ curve in terms of CFU (no log-transformation of raw data), with $\varepsilon_{i j}$ the common errors term having a mean of 0 and a variance $\sigma_{1}^{2}$.

When there is only one exponential error model, the predictions and observations are automatically log-transformed by Phoenix and fitted in that space, so that the error model was actually a Log-additive error model.

Parameter estimates, with their associated SE and CV as a measure of precision, were based on minimizing an objective function value, using Laplace engine for the Maximum Likelihood Estimation.

For P. multocida, there were no values reported as below the quantification limit (BQL) due to some re-growth at $24 \mathrm{~h}$. For M. haemolytica, data reported as BQL (7\% of the data set) were retained in the analysis by using a likelihood-based approach according to the M3 method (Beal, 2001). Diagnostic plots determined whether the model was adequate: these included PRED (population (zero-eta) prediction) and IPRED (individual prediction) versus the dependent variable, Conditional weighted residuals (CWRES) and individual fitting. The overall adequacy of the model was established by plotting the Visual Predictive Check (VPC) i.e., a graphical comparison between the observed data and prediction intervals (20-80th percentiles) derived from the simulated data (data set simulated 500 times).

Secondary parameters computed were MIC and minimal bactericidal concentration (MBC). MIC and MBC indicate AMD PD parameters (efficacy, potency, sensitivity) but also test tube conditions [growth and death rates, duration of observation (often 18-24 h) and the initial inoculum load (usually $5 \times 10^{5}$ $\mathrm{CFU} / \mathrm{mL}$ )]. According to Mouton and Vinks (2005), MIC is related to the aforementioned factors by eqs. $6 \mathrm{~A}, \mathrm{~B}$ :

$$
M I C=E C_{50} \times\left(\frac{K_{G R O W T H}-0.29}{E_{M A X}-\left(K_{G R O W T H}-0.29\right)}\right)^{\frac{1}{\text { Gamma }}}
$$

where $K_{G R O W T H}$, (actually $K_{G R O W T H}-K_{D E A T H}$ ) (for present data it is $K_{\text {GROWTHMAX }}$ ), EC $50, E_{\mathrm{MAX}}$, and Gamma as defined above;

Time of measurement was fixed at $18 \mathrm{~h}$ and it is assumed that visible growth indicates an inoculum of $1 \times 10^{8} \mathrm{CFU} / \mathrm{mL}$; hence, the constant 0.29 of eq. $6 \mathrm{~A}$ is obtained from eq. $6 \mathrm{~B}$ :

$$
\frac{1}{\text { Time of measurement }(18 h)} \times \operatorname{LN}\left(\frac{N(t)}{N(0)}\right)=0.294
$$

where $N(\mathrm{t})$ is the inoculum size at $18 \mathrm{~h}$ i.e., $10^{8} \mathrm{CFU} / \mathrm{mL}$ and $N(0)$ is the initial inoculum i.e., $5 \times 10^{5} \mathrm{CFU} / \mathrm{mL}$. When the initial load is not $5 \times 10^{5} \mathrm{CFU} / \mathrm{mL}$ as for the Sidhu et al. (2014), data eq. $6 \mathrm{~B}$ should be edited to replace 0.29 by the ad hoc value; for example, using an initial count of $10^{7} \mathrm{CFU} / \mathrm{mL}$, the constant is no longer 0.29 but -0.127 . 
Similarly, $\mathrm{MBC}$ is computed by replacing, in the previous equation, $10^{8}$ by $5 \times 10^{2} \mathrm{CFU} / \mathrm{mL}$; MBC corresponds to at least 99.9\% kill from the initial inoculum $\left(5 \times 10^{5} \mathrm{CFU} / \mathrm{mL}\right)$ (Mouton and Vinks, 2005); MBC is given by eq. 7:

$$
M B C=E C_{50} \times\left(\frac{K_{G R O W T H}+0.383}{E_{M A X}-K_{G R O W T H}+0.383}\right)^{\frac{1}{\text { Gamma }}}
$$

The Phoenix model code is available on request and will be made available by the authors, without undue reservation, to any qualified researcher.

The estimated fixed parameters $\left(\mathrm{E}_{M A X}, \mathrm{EC}_{50}\right.$, Gamma, $\left.\mathrm{K}_{\text {GROWTHMAX }}, \mathrm{K}_{D E A T H}, \mathrm{~B}_{M A X}\right)$ and Alpha were reported as typical values with coefficient of variation.

\section{Simulation of Two Possible Dosing Regimens and in silico Dose Fractionation to Select a PK/PD Index for Florfenicol}

Selection of the best PK/PD index for florfenicol and its magnitude were calculated using the in silico PK/PD model, simulating several dosage regimens using eq. 2 with $C(t)$ being the predicted plasma florfenicol concentration obtainable in vivo. $C(t)$ was determined by solving the population $\mathrm{PK}$ model developed for florfenicol in calves by Toutain et al. (2017), which is a meta-analysis of PK studies in which calves were administered $40 \mathrm{mg} / \mathrm{kg}$ of florfenicol subcutaneously (300 $\mathrm{mg} / \mathrm{mL}$ Solution for Injection). The design of the population pharmacokinetic study and the resulting estimated PK parameters used for these simulations are presented in Supplementary File S3. Using the PK/PD in silico model, the microbiological effect of two possible licensed dosing regimens were simulated: single dose $(40 \mathrm{mg} / \mathrm{kg})$ versus $20 \mathrm{mg} / \mathrm{kg}$ twice at $48 \mathrm{~h}$ dosing interval. Using the same PK/PD model, dose fractionation was conducted for doses of $0,2.5,5,10,20$, 30, 40 (licensed dose), 50, 60, and $80 \mathrm{mg} / \mathrm{kg}$ given, as a single administration, two administrations at $48 \mathrm{~h}$ interval or 4 administrations at $24 \mathrm{~h}$ intervals, yielding a total of 28 possible exposure patterns. Simulations were performed for two initial loads $\left(10^{5}\right.$ and $\left.10^{7} \mathrm{CFU} / \mathrm{mL}\right)$ and for four MIC levels $(0.5,1,2$, and $4 \mathrm{mg} / \mathrm{L})$. We assumed that differences in MIC were due solely to altered potency and not efficacy. For simulation at MICs of $0.5,1,2$, and $4 \mathrm{mg} / \mathrm{L}$, the $\mathrm{EC}_{50}$ fitted from TKCs was multiplied by a scaling factor converting measured MIC $(0.4 \mathrm{mg} / \mathrm{L}$ for $P$. multocida and $0.5 \mathrm{mg} / \mathrm{L}$ for M. haemolytica) to the simulated MIC. For the bacteriological response, the cumulative Area Under the Curve of the total bacterial count over $96 \mathrm{~h}\left(\mathrm{AUC}_{\mathrm{bact}} 96 \mathrm{~h}\right)$ was used. Data were then $\log 10$ transformed for regression. When the bacterial count had decreased to $30 \mathrm{CFU} / \mathrm{mL}$, it was considered that regrowth would not occur and curves were truncated for this cut-off value. PK/PD indices are conventionally determined using plasma protein unbound (free) concentration. The latest study at the time of writing reported that florfenicol protein binding was only $5 \%$ at the high concentration and was negligible at the low concentrations, representing a $f u$ of essentially 1.0 (Foster et al., 2016). We therefore hypothesized that the binding of florfenicol to plasma protein could be ignored and that we simulated free plasma concentrations in the dose fractionation (vide infra). The area under the plasma concentration-time curve $\left(f \mathrm{AUC}_{\mathrm{PK}}(0-96 \mathrm{~h})\right.$ ) and percentage time plasma concentration exceeded MIC within $96 \mathrm{~h}(f \mathrm{~T}>\mathrm{MIC} \%)$ were computed using the statistical tool of Phoenix. The 28 pairs of $f \mathrm{~T}>\mathrm{MIC} \%$ (independent variable) versus $\mathrm{AUC}_{\mathrm{bact} 96 \mathrm{~h}}$ (dependent variable) and $f \operatorname{AUC}_{\mathrm{PK}}(0-96 \mathrm{~h}) / \mathrm{MIC}$ (independent

TABLE 1 | Primary and secondary parameter estimates and precision (CV\%).

\begin{tabular}{|c|c|c|c|c|c|}
\hline \multirow[b]{2}{*}{ Primary parameters $^{a}$} & \multirow[b]{2}{*}{ Unit } & \multicolumn{2}{|c|}{ Pasteurella multocida } & \multicolumn{2}{|c|}{ Mannheimia haemolytica } \\
\hline & & Estimate & CV\% & Estimate & CV\% \\
\hline \multicolumn{6}{|c|}{ Bacteria growth system parameters } \\
\hline KGROWTHMAX & $1 / \mathrm{h}$ & 0.97 & 5.1 & 1.58 & 10.7 \\
\hline $\mathrm{K}_{\mathrm{DEATH}}$ & $1 / \mathrm{h}$ & 0.12 & 11.9 & 0.78 & 11.1 \\
\hline$B_{\text {MAX }}$ & $\mathrm{CFU} / \mathrm{mL}$ & $5.2 \times 10^{9}$ & 38.1 & $9.6 \times 10^{8}$ & 34.6 \\
\hline alpha & $1 / \mathrm{h}$ & 0.22 & 8.9 & 0.93 & 12.8 \\
\hline \multicolumn{6}{|c|}{ Florfenicol pharmacodynamic parameters } \\
\hline $\mathrm{EC}_{50}$ & $\mathrm{mg} / \mathrm{L}$ & 0.46 & 7.5 & 0.70 & 7.2 \\
\hline gamma & scalar & 2.74 & 8.9 & 2.63 & 11.9 \\
\hline $\mathrm{E}_{\mathrm{MAX}}$ (maximal killing rate) & $1 / \mathrm{h}$ & 2.00 & 2.4 & 2.70 & 8.4 \\
\hline stdev0 (Ln domain) & scalar & $1.11(156 \mathrm{CV} \%)$ & 9.9 & 1.05 (141 CV\%) & 5.5 \\
\hline \multicolumn{6}{|l|}{ Secondary parameters ${ }^{b}$} \\
\hline MIC (10 5 CFU/mL inoculum) & $\mathrm{mg} / \mathrm{L}$ & 0.35 & 5.9 & 0.43 & 8.1 \\
\hline MIC (10 CFU/mL inoculum) & $\mathrm{mg} / \mathrm{L}$ & 0.40 & 6.4 & 0.49 & 7.1 \\
\hline stationary concentration & $\mathrm{mg} / \mathrm{L}$ & 0.41 & 6.5 & 0.50 & 7.0 \\
\hline $\mathrm{MBC}$ & $\mathrm{mg} / \mathrm{L}$ & 0.51 & 7.5 & 0.60 & 6.3 \\
\hline
\end{tabular}

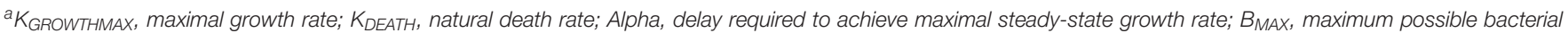

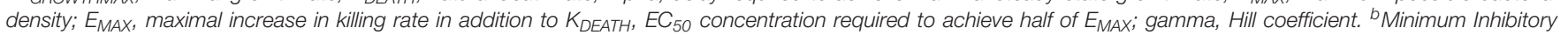
(MIC) and Bactericidal (MBC) concentrations and stationary concentrations computed according to Mouton and Vinks (6). 
variable) versus $\mathrm{AUC}_{\text {bact96h }}$ (dependent variable) obtained for each MIC were fitted with an Inhibitory Effect Sigmoid $I_{\max }$ PD model (Model 108), Eq. 8:

$$
\text { Effect }=E_{0}-\left(\frac{I_{\max } \times I N D E X^{\text {Slope }}}{I N D E X^{\text {Slope }}+I N D E X_{50}^{\text {Slope }}}\right)
$$

where $\mathrm{E}_{0}$ is the maximum effect (obtained for the control curve for $C(t)=0)$, the maximum possible observed effect is $\left(E_{0}-I_{\max }\right)$, $I_{\text {max }}$ being the amplitude of maximal effect. INDEX $X_{50}$ is the magnitude of the index $\left(f A U C_{P K}(0-96 \mathrm{~h}) / M I C\right.$ or $\left.f T>M I C \%\right)$ that achieves $50 \%$ of the $I_{\max }$, and Slope is the sigmoidicity factor, reflecting the steepness of the relationship. Curve fitting was performed with WinNonlin ${ }^{\circledR}$ using the non-linear least-squares algorithm. The coefficients of determination $\left(R^{2}\right)$, the Akaike Information Criterion (AIC), and visual inspection of graphs were used to select the PK/PD index that best predicted the antibacterial effect. The INDEX $X_{90 \%}$ was computed as the breakpoint value of the predicting PK/PD index.

\section{RESULTS}

\section{Time-Kill Curve Modeling}

Parameter estimates from the TKC model (bacteria growth system and drug sub-models) are summarized in Table 1. The precision of the estimation of the parameter value was good in all cases (estimated CV\% less than 38\%).

Maximal growth rate (K $\mathrm{K}_{\mathrm{GROWTHMAX}}$, per hour) was 0.97 for $P$. multocida (yielding a $0.71 \mathrm{~h}$ generation half-life) and 1.58 for $M$. haemolytica (yielding a $0.44 \mathrm{~h}$ generation half-life). The natural death rate ( $\mathrm{K}_{\mathrm{DEATH}}$, per hour) was 0.12 for P. multocida (yielding a $5.9 \mathrm{~h}$ count-halving half-life) and 0.78 for $M$. haemolytica (yielding a $0.9 \mathrm{~h}$ count-halving half-life). The delay in achieving a maximal steady-state growing rate (Alpha) was $0.22 \mathrm{~h}^{-1}$ for P. multocida and $0.93 \mathrm{~h}^{-1}$ for M. haemolytica, corresponding to half-lives to establish full growth capacity of 3 and $0.75 \mathrm{~h}$, respectively. The maximum possible bacterial density of the cultures ( $\mathrm{B}_{\mathrm{MAX}}$ ) was $5.2 \times 10^{9} \mathrm{CFU} / \mathrm{mL}$ for P. multocida and $9.6 \times 10^{8} \mathrm{CFU} / \mathrm{mL}$ for $M$. haemolytica. The CV\% for inter-strain (assay) variability was $89 \%$ for P. multocida and $430 \%$ for $M$. haemolytica. Low values of eta-shrinkage (12\% P. multocida and $4 \%$ for $M$. haemolytica) confirm the identifiability of the random effect on $\mathrm{B}_{\max }$.

The maximal drug-induced increase in bacterial killing rate ( $\mathrm{E}_{\max }$, per hour) was $2.0 \mathrm{~h}^{-1}$ for $P$. multocida (yielding a 16.7-fold increase in overall death rate) and $2.7 \mathrm{~h}^{-1}$ for M. haemolytica (yielding a 3.5-fold increase in overall death rate). The in vitro concentration for achieving half the maximal effect $\left(\mathrm{EC}_{50}\right)$ was $0.46 \mathrm{mg} / \mathrm{L}$ for $P$. multocida and $0.70 \mathrm{mg} / \mathrm{L}$ for M. haemolytica, ranking favorably for average experimental MICs of $0.4 \mathrm{mg} / \mathrm{L}$ for P. multocida and $0.5 \mathrm{mg} / \mathrm{L}$ for M. haemolytica. The slope of the concentration-effect curve (gamma, dimensionless scalar) was for 2.74 for P. multocida and 2.63 for M. haemolytica.

The plot of the observed natural logarithm of bacterial counts (CFU/mL, the dependent variable DV) versus individual predicted count values (IPRED) for $P$. multocida and
M. haemolytica is presented in Figure 2. Visual predictive check (VPC) for P. multocida and M. haemolytica are shown in Figure 3.

\section{Comparison of Microbiological Response for Two Possible Modalities of Florfenicol Administration}

The in silico predicted microbiological efficacy of the two approved dosage regimens for florfenicol were similar for two inoculum sizes (low $10^{5}$ and high $10^{7} \mathrm{CFU} / \mathrm{mL}$ ) for P. multocida and $M$. haemolytica at MICs of $0.5,1,2$, and $4 \mathrm{mg} / \mathrm{L}$ (Figure 4). For an MIC of $2 \mathrm{mg} / \mathrm{L}$, the single administration of $40 \mathrm{mg} / \mathrm{kg}$ was clearly superior to the two administrations of $20 \mathrm{mg} / \mathrm{kg}$ at $48 \mathrm{~h}$ interval for both $P$. multocida and M. haemolytica and with both inoculum counts. For an MIC of $4 \mathrm{mg} / \mathrm{L}$, none of the dosage regimens were predicted to be efficacious by the in silico PK/PD model.

\section{Dose Fractionation in silico}

Figure 5 illustrates the fitting comparison for prediction of $\left.\log 10 \mathrm{AUCbact}_{(0-96 \mathrm{~h}}\right) \quad\left(\mathrm{I}_{\max }\right.$ sigmoid model) using $\mathrm{fAUC}_{(\mathrm{PK} 0-96 \mathrm{~h})} / \mathrm{MIC}$ or $f \mathrm{~T}>\mathrm{MIC} \%$ as the predictive variable for MICs $0.5,1,2$, and $4 \mathrm{mg} / \mathrm{L}$ and for inoculum strengths of $10^{5}$ and $10^{7} \mathrm{CFU} / \mathrm{mL}$ for both $P$. multocida and M. haemolytica. The fitting for MIC $4 \mathrm{mg} / \mathrm{L}$ was excluded due to the limited efficacy of even the highest dosage regimen. In all cases, $f$ AUC/MIC was a better PK/PD index than $f \mathrm{~T}>$ MIC over $96 \mathrm{~h}$. For P. multocida, the goodness of fit values, averaged for inoculum sizes of $10^{5}$ and $10^{7} \mathrm{CFU} / \mathrm{mL}$ and for all MICs, were better for $f \mathrm{AUC}_{\mathrm{PK}}(0-96 \mathrm{~h}) / \mathrm{MIC}\left(\mathrm{AIC}=76.9, R^{2}=0.939\right)$ than for $f \mathrm{~T}>\mathrm{MIC}\left(\mathrm{AIC}=81.3, R^{2}=0.934\right)$. For $M$. haemolytica, the goodness of fit values, averaged for inoculum sizes of $10^{5}$ and $10^{7} \mathrm{CFU} / \mathrm{mL}$ and for all MICs, were also better for $f \mathrm{AUC}_{\mathrm{PK}(0-96 \mathrm{~h})} / \mathrm{MIC}\left(\mathrm{AIC}=84, R^{2}=0.924\right)$ than for $f \mathrm{~T}>\mathrm{MIC}$ $\left(\mathrm{AIC}=86.3, R^{2}=0.924\right)$.

The critical value for $90 \%$ of the maximal in silico possible anti-bacterial action for $f \mathrm{AUC}_{\mathrm{PK}}(0-96 \mathrm{~h}) / \mathrm{MIC}$ was solved using Eq. 8 for the two inoculum strengths actually tested $\left(10^{5}\right.$ and $10^{7}$ CFU/mL) and for MICs of $0.5,1$, and $2 \mathrm{mg} / \mathrm{L}$ (Table 2).

Data in Table 2 indicate that the critical value of the PK/PD index $\left(f \mathrm{AUC}_{\mathrm{PK}}(0-96 \mathrm{~h}) / \mathrm{MIC}\right)$ to achieve $90 \%$ of maximal effect) was dependent of the tested MIC but relatively similar for both bacterial species. For a MIC of $1 \mathrm{mg} / \mathrm{L}$, the critical value for fAUC $_{\mathrm{PK}(0-96 \mathrm{~h})} / \mathrm{MIC}$ for P. multocida was 115 and $134 \mathrm{~h}$ for inocula of $10^{5}$ and $10^{7} \mathrm{CFU} / \mathrm{ml}$, respectively. The corresponding values were 127 and $133 \mathrm{~h}$ for $M$. haemolytica. These values indicate that, to achieve $90 \%$ of maximal efficacy for a pathogen having a MIC of $1 \mathrm{mg} / \mathrm{L}$, the average free plasma florfenicol concentration over $96 \mathrm{~h}$ should be equal to 1.19 - and 1.32fold the MIC for P. multocida and 1.40 and 1.39 fold the MIC for M. haemolytica.

\section{DISCUSSION}

This study is the first to quantify, for veterinary pathogens, the three basic PD parameters of an AMD from TKC analysis, 


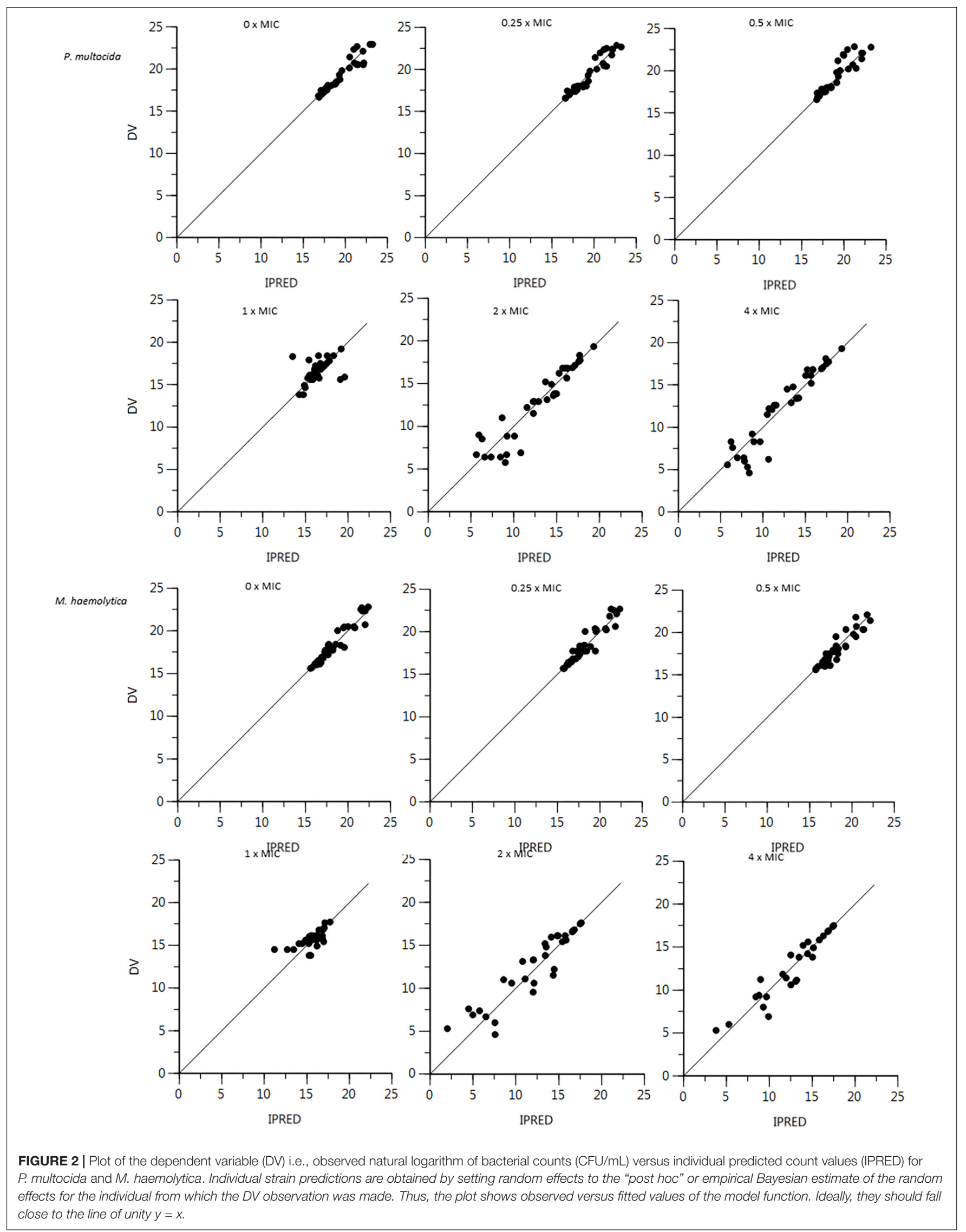




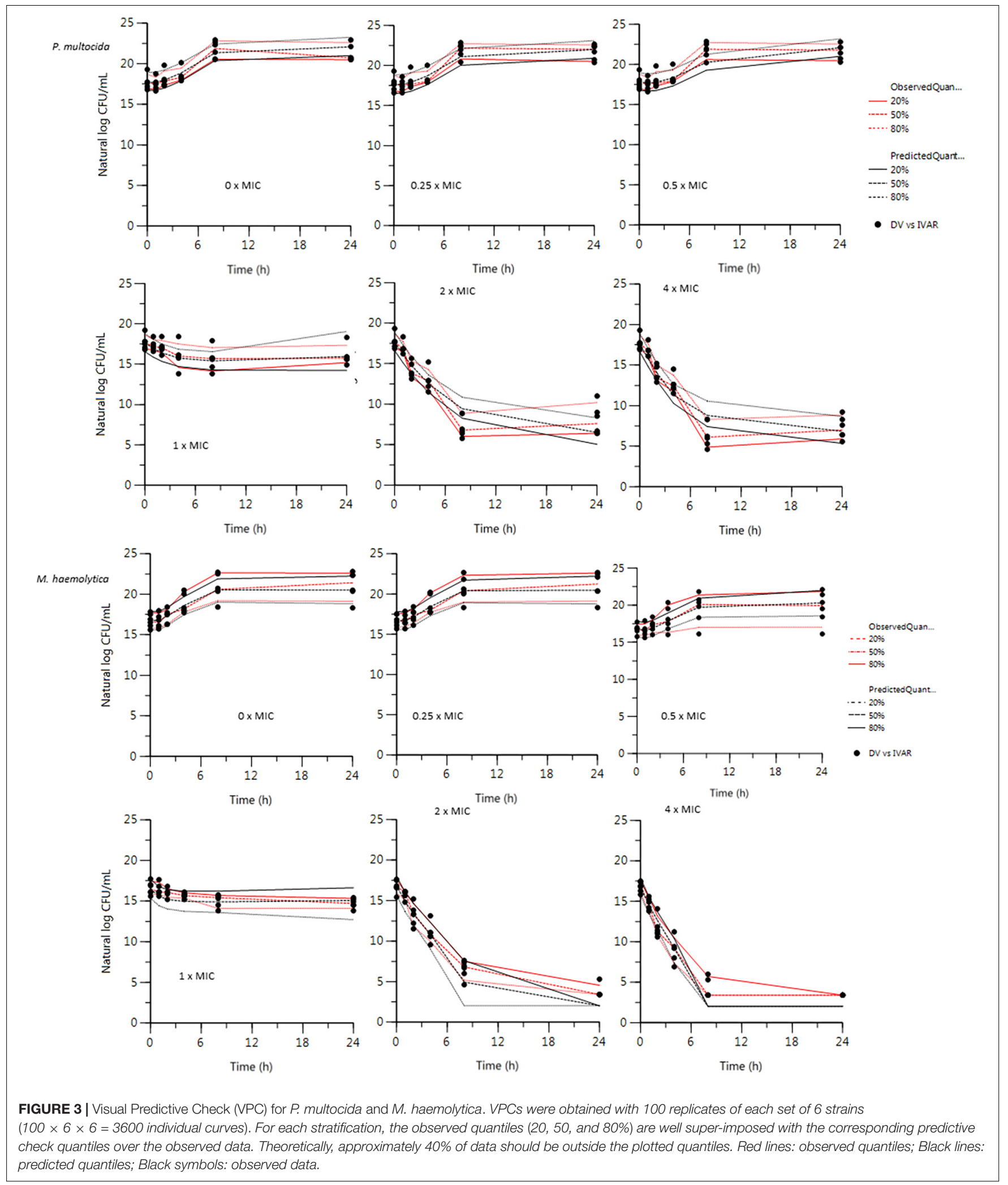

namely efficacy ( $\mathrm{E}_{\mathrm{MAX}}$ maximum killing rate), potency $\left(\mathrm{EC}_{50}\right)$ and sensitivity (slope of the concentration-effect relationship). These data have been obtained for florfenicol and two major calf pathogens, P. multocida and M. haemolytica. The classical index describing quantitatively AMD action is MIC. However, MIC is not a genuine PD parameter; it is a reproducible hybrid 


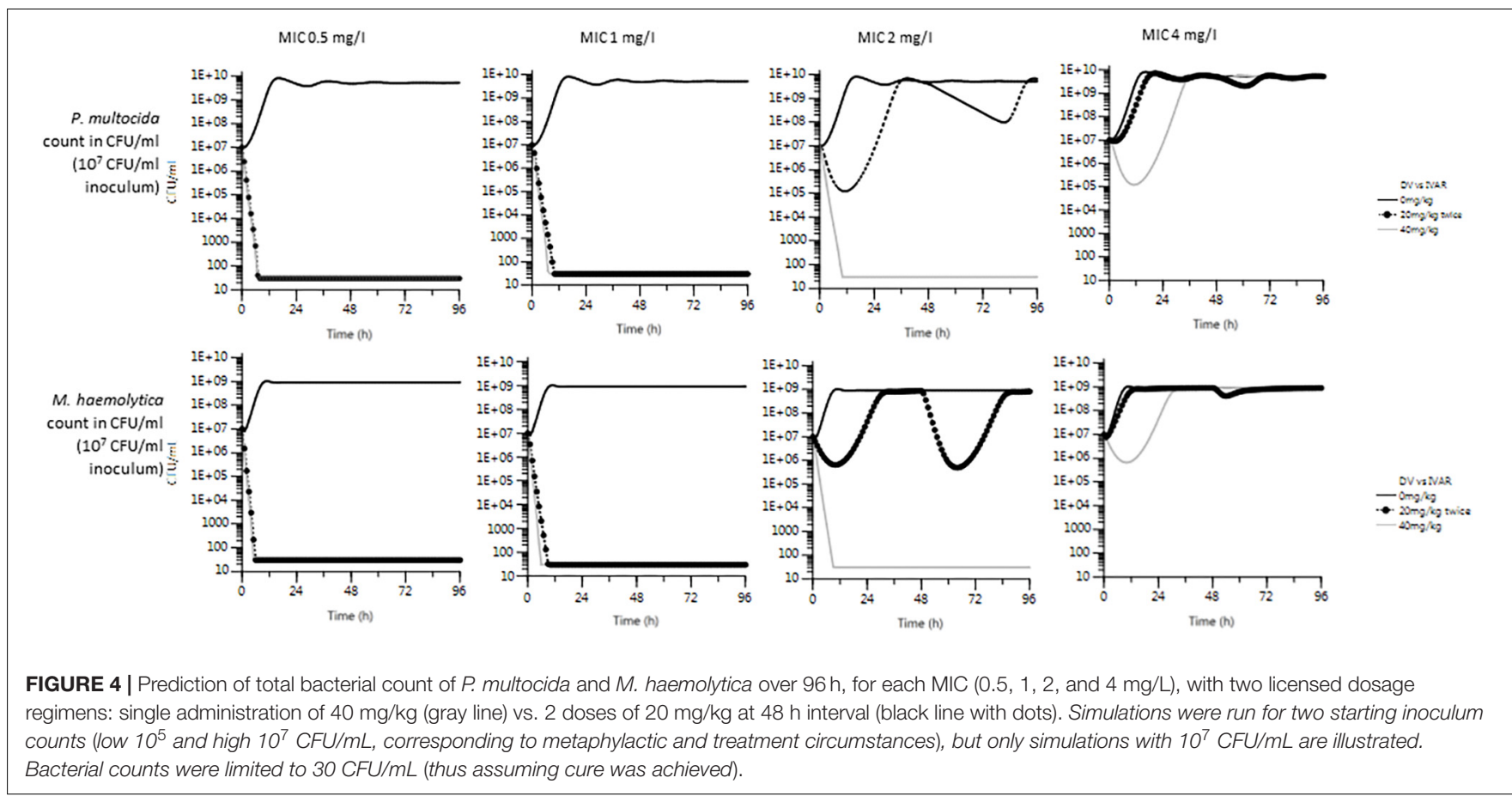

variable measured under standard conditions. Actually, MIC is dependent not only on the three PD parameters but also on in vitro conditions (growth and death rates of the tested pathogen, duration of observation and the initial inoculum load). The numerical value of each MIC therefore depends on seven separate factors, as explicitly indicated in eqs. 6A,B.

The advantage of dissecting MIC into these dependency components is to identify the test tube conditions that can be regarded as confounding factors from the actual PD properties that are of primary interest, not only for AMDs but for drugs of all classes. The three parameters have been dissected out and quantified by modeling TKC data. In contrast with MIC, as a crude index of AMD action, TKCs describe time course as well as magnitude of antibacterial action over the 18-24 h duration of exposure. This enables capture of the pattern of bacterial killing with semi-mechanistic models of the type used in the present paper (Figure 1). This model has recently been evaluated against similar PK/PD models proposed by others and using Monte Carlo Simulations. It was concluded that, under constant drug concentrations, as in this study, the median PD parameter estimates were within $10 \%$ of the true value and the precision was $<20 \%$ (Jacobs et al., 2016).

The calculations indicate that potency and efficacy of florfenicol were of the same order of magnitude for the two pathogens investigated. The strains belonged to the distribution of the wild population for the two pathogens as EUCAST epidemiological cut-off values (ECOFFs) values are 1 and $2 \mathrm{mg} / \mathrm{L}$ for P. multocida and M. haemolytica, respectively. In future studies, it would be valuable to subject to the same modeling process strains belonging to resistant sub-populations; this would reveal how resistance is phenotypically expressed (for example, as either an increase in $\mathrm{EC}_{50}$ and/or a reduction in $\mathrm{E}_{\mathrm{MAX}}$ ). Such data would enable interpretation of mechanisms of emergence of resistance, using the same conceptual framework for drugs of other pharmacological classes, when analyzing drug-receptor interaction. Such analysis is a major tool in the quest for developing new drugs (Kenakin, 1997).

The analysis presented in this paper adds a new dimension to bactericidal killing curves by converting them into proxies of an infection model. This required linking in vivo PK data to a PD TKC model able to predict the temporal dynamics of bactericidal activity. The PK data are generated by solving a model readily obtained through either classical or population investigations. The PD model predicts a microbiological response for a given drug exposure at two inoculum levels of $10^{5}$ and $10^{7} \mathrm{CFU} / \mathrm{mL}$ (corresponding to metaphylactic and treatment circumstances, respectively). Currently, rodent models are widely used but they raise questions of cost and ethical use of animals in research. As an alternative to animal studies, the hollow fiber model has been developed as a dynamic infection model (Michael et al., 2014) but its use in veterinary medicine has not yet been reported. Hollow fiber technology is costly and resource demanding; few alternatives like chemostats can be explored but have their own limitations. The present adaptation of TKC results offers the advantages of using historical data and its availability for many veterinary pathogens. Hence, data meta-analysis, as presented in this article, provides, at low cost and with benefits for animal ethics, a new approach to selection of a PK/PD index to predict clinical efficacy of AMDs used in veterinary medicine.

The selected PD model simulated the time course of bactericidal activity of florfenicol, with pathogen exposure 


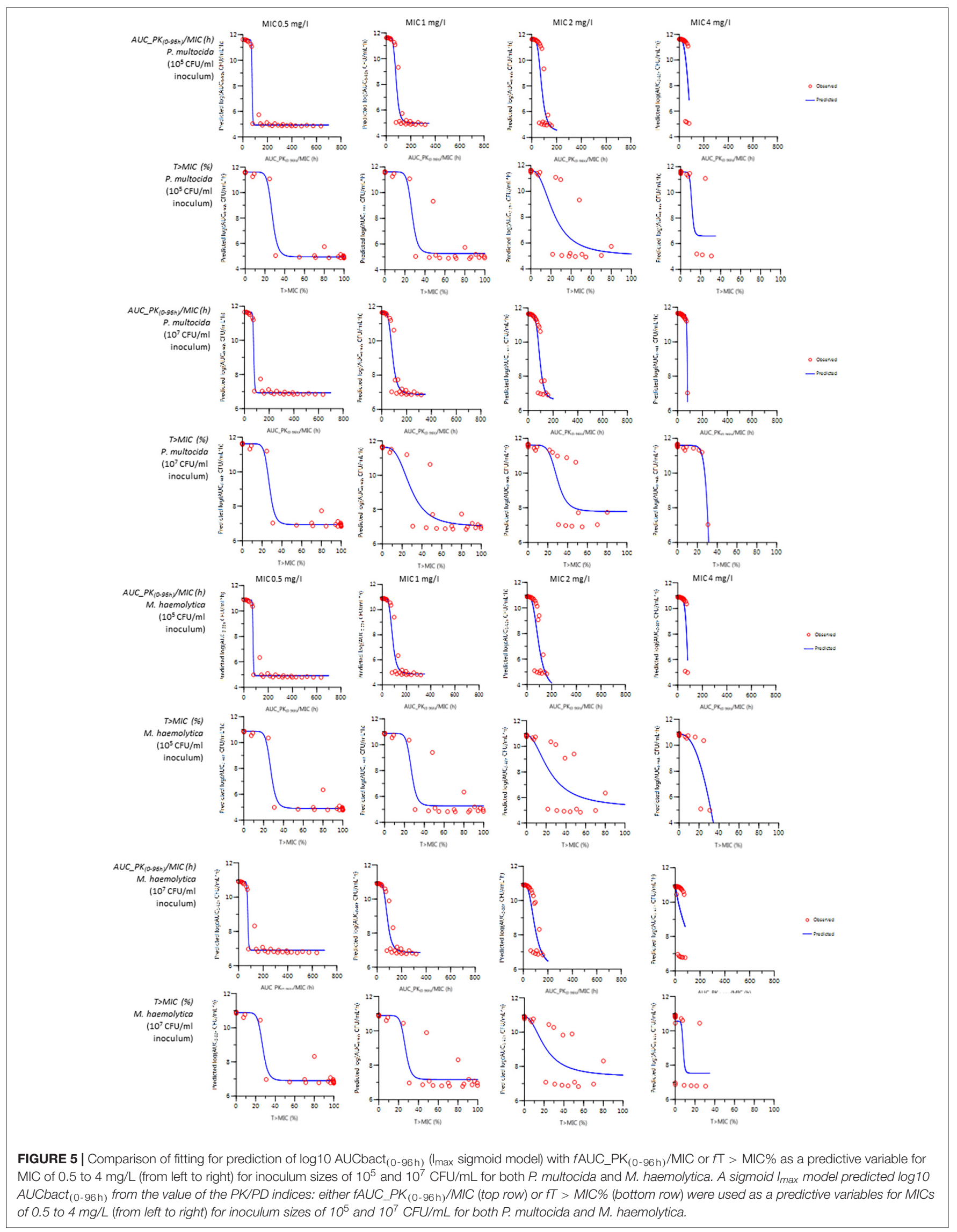


TABLE 2 | Critical value of the PK/PD index (fAUC $\mathrm{PK}_{(0-96 \mathrm{~h})} / \mathrm{MIC}$, unit h) to achieve $50 \%$ or $90 \%$ of the maximal possible in silico bacteriological effect.

\begin{tabular}{|c|c|c|c|c|c|c|c|}
\hline \multicolumn{2}{|c|}{ Efficacy (\% Emax) } & \multicolumn{2}{|c|}{ P. multocida } & \multicolumn{2}{|c|}{ M. haemolytica } & \multirow{2}{*}{\multicolumn{2}{|c|}{$\begin{array}{l}\text { Average free plasma concentration required over } 96 \mathrm{~h} \\
\text { (expressed in MIC-fold, unitless) to achieve } 90 \% \text { of } \\
\text { the maximal efficacyb }\end{array}$}} \\
\hline & \multirow[b]{2}{*}{ MIC (mg/L) } & $50 \%$ & $90 \%$ & $50 \%$ & $90 \%$ & & \\
\hline & & & fAUC $_{P K}$ & h) $/ \mathrm{MIC}$ & & P. multocida & M. haemolytica \\
\hline \multirow[t]{3}{*}{$10^{5} \mathrm{CFU} / \mathrm{mL}$ inoculum } & 0.5 & 75 & 81 & 75 & 81 & 0.84 & 0.84 \\
\hline & 1 & 81 & 115 & 83 & 127 & 1.19 & 1.32 \\
\hline & 2 & 79 & 133 & 94 & 186 & 1.38 & 1.93 \\
\hline \multirow[t]{3}{*}{$10^{7} \mathrm{CFU} / \mathrm{mL}$ inoculum } & 0.5 & 75 & 84 & 75 & 83 & 0.87 & 0.86 \\
\hline & 1 & 85 & 134 & 84 & 133 & 1.40 & 1.39 \\
\hline & 2 & 92 & 137 & 94 & 195 & 1.43 & 2.03 \\
\hline
\end{tabular}

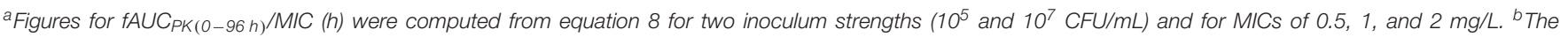

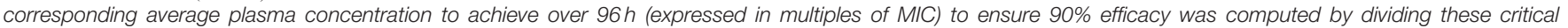
values by $96 \mathrm{~h}$. Bold fonts highlight values for MIC of $1 \mathrm{mg} / \mathrm{L}$.

actually obtained in vivo after administration to calves of the reference florfenicol formulation $\left(\mathrm{Nuflor}^{\circledR}\right)$. To achieve this, the PD component of the model with its estimated parameters was solved using plasma florfenicol concentrations as predicted by a florfenicol population model (Supplementary File S3, also see Toutain et al., 2019).

Thus, several florfenicol exposure scenarios were simulated to generate corresponding killing curves. This leads to the conclusion that a single florfenicol dose of $40 \mathrm{mg} / \mathrm{kg}$ should be more efficacious in bactericidal effect than an alternative dosing regimen of two $20 \mathrm{mg} / \mathrm{kg}$ dose at a $48 \mathrm{~h}$ interval. According to a meta-analysis from DeDonder and Apley (2015), both dosage regimen were equally efficacious (absolute risk reduction of morbidity) versus negative control.

In order to propose a PK/PD breakpoint for florfenicol based on the VetCAST approach, the first step is to select an appropriate $\mathrm{PK} / \mathrm{PD}$ index predicting efficacy. A PK/PD approach is superior to using a target CFU at $24 \mathrm{~h}$ as it allows the description of the onset, rate and extent of killing and a data-based determination as to whether an AMD is time or concentration-dependent. Florfenicol is used solely in veterinary medicine, so that historically no dose fractionation rodent studies are available to determine the most appropriate PK/PD index predicting efficacy. For determination of the best PK/PD index, in silico simulation approaches are scientifically attractive, ethically acceptable and low cost alternatives to in vivo dose fractionation studies. This in silico approach has been validated for human medicine for the main AMD classes (Nielsen and Friberg, 2013). To select $f$ AUC/MIC or $f \mathrm{~T}>\mathrm{MIC}$ as the PK/PD index of choice, it is necessary to establish the influence of both level (concentration) and shape of exposure to florfenicol on the efficacy of its bactericidal effect, as predicted by the PD model. In this study, from simulated killing curves obtained with 10 florfenicol dose levels ranging from 0 to $80 \mathrm{mg} / \mathrm{kg}$ and divided into one, two or four administrations at differing dosing intervals, 28 killing curve profiles were generated. These were then modeled using the classical $\mathrm{E}_{\max }$ model, with the $\mathrm{PK} / \mathrm{PD}$ index as independent variable and $\mathrm{fAUC}_{(0-96 \mathrm{~h})}$ under the killing curves as dependent variable. For MICs of $0.5,1$, and $2 \mathrm{mg} / \mathrm{L}, \mathrm{fAUC} / \mathrm{MIC}$ was systematically superior to $f \mathrm{~T}>\mathrm{MIC}$ in predicting bacterial killing, although for the lowest MIC $(0.5 \mathrm{mg} / \mathrm{L})$ both indices were acceptable. For an MIC of $2 \mathrm{mg} / \mathrm{L}$, the relationship degraded for $f \mathrm{~T}>\mathrm{MIC}$ but remained acceptable for $f$ AUC/MIC. For an MIC of $4 \mathrm{mg} / \mathrm{L}$, both indices failed to predict adequately the florfenicol response or lack thereof.

The selection of $f$ AUC/MIC as the best PK/PD index for florfenicol is consistent with a previous report that, regardless of AMD class, $f \mathrm{AUC} / \mathrm{MIC}$ is the most appropriate index when terminal half-life is long (Nielsen and Friberg, 2013).

In this study, results of simulations are presented using free plasma concentration of florfenicol, as free plasma concentration is the best proxy for concentration in the biophase. In non-lactating dairy cattle, plasma protein binding ranged from 19 to $23 \%$ (Bretzlaff et al., 1987). However, a recent investigation reported that the degree of florfenicol binding in 6-month old steers was either very low (5\%) or negligible (Foster et al., 2016). Such low binding differs from another recent study (Mzyk et al., 2018). Investigating the influence of age (1 to 168 days) on degree of florfenicol plasma protein binding, these authors reported binding ranging from 12 to $42 \%$ in one-day old, and from 11 to $32 \%$ in 168-day old animals, at a concentration of $1 \mathrm{mg} / \mathrm{L}$. In light of these inter-study differences, and as the selected PK/PD index is $f$ AUC/MIC, it would be a simple matter to apply a correction for unbound fraction during the computation of the PK/PD cut-off for florfenicol by Monte Carlo simulation. On this basis, it is concluded that for both P. multocida and M. haemolytica maximum efficacy (actually 90\%) over $96 \mathrm{~h}$ is obtained when the average free plasma concentration is equal to the 1.2 to 1.4 times the respective MIC.

\section{DATA AVAILABILITY}

All datasets analyzed for this study are included in the manuscript and the Supplementary Files S1-S3.

\section{AUTHOR CONTRIBUTIONS}

PL and PS generated raw data. LP retrieved and validated raw data. P-LT and LP performed the modeling analysis and 
drafted the manuscript. All authors critically reviewed several drafts of the manuscript.

\section{FUNDING}

This work was partly supported by the Direction Générale de l'Alimentation (DGAL) of the French Ministry of Agriculture and Food. DGAL has no role in data collection, interpretation and the decision to submit this work for publication.

\section{REFERENCES}

Beal, S. L. (2001). Ways to fit a PK model with some data below the quantification limit. J. Pharmacokinet. Pharmacodyn. 28, 481-504.

Bretzlaff, K. N., Neff-Davis, C. A., Ott, R. S., Koritz, G. D., Gustafsson, B. K., and Davis, L. E. (1987). Florfenicol in non-lactating dairy cows: pharmacokinetics, binding to plasma proteins, and effects on phagocytosis by blood neutrophils. J. Vet. Pharmacol. Ther. 10, 233-240. doi: 10.1111/j.1365-2885.1987.tb 00534.x

DeDonder, K. D., and Apley, M. D. (2015). A review of the expected effects of antimicrobials in bovine respiratory disease treatment and control using outcomes from published randomized clinical trials with negative controls. Vet. Clin. North Am. Food Anim. Pract. 31, 97-111. doi: 10.1016/j.cvfa.2014.11.003

Foster, D. M., Martin, L. G., and Papich, M. G. (2016). Comparison of active drug concentrations in the pulmonary epithelial lining fluid and interstitial fluid of calves injected with enrofloxacin, florfenicol, ceftiofur, or tulathromycin. PLoS One 11:e0149100. doi: 10.1371/journal.pone.0149100

Jacobs, M., Gregoire, N., Couet, W., and Bulitta, J. B. (2016). Distinguishing antimicrobial models with different resistance mechanisms via population pharmacodynamic modeling. PLoS Comput. Biol. 12:e1004782. doi: 10.1371/ journal.pcbi.1004782

Kenakin, T. P. (1997). Pharmacologic Analysis of Drug-Receptor Interaction, 3rd Edn. Philadelphia: Lippincott-Raven Publishers.

Lees, P., Pelligand, L., Illambas, J., Potter, T., Lacroix, M., Rycroft, A., et al. (2015). Pharmacokinetic/pharmacodynamic integration and modelling of amoxicillin for the calf pathogens Mannheimia haemolytica and Pasteurella multocida. J. Vet. Pharmacol. Ther. 38, 457-470. doi: 10.1111/jvp.12207

Michael, J., Barth, A., Kloft, C., and Derendorf, H. P. (2014). "Pharmacodynamic in vitro models to determine the effect of antibiotics," in Fundamentals of Antimicrobial Pharmacokinetics and Pharmacodynamic, eds D. H. Vinks and A. Mouton (New York, NY: Springer), 81-112. doi: 10.1007/978-0-387-75613-4_5

Mouton, J. W., and Vinks, A. A. (2005). Relationship between minimum inhibitory concentration and stationary concentration revisited: growth rates and minimum bactericidal concentrations. Clin. Pharmacokinet. 44, 767-768. doi: 10.2165/00003088-200544070-00007

\section{ACKNOWLEDGMENTS}

The VetCAST steering committee is acknowledged for the support to this publication.

\section{SUPPLEMENTARY MATERIAL}

The Supplementary Material for this article can be found online at: https://www.frontiersin.org/articles/10.3389/fmicb. 2019.01237/full\#supplementary-material

Mzyk, D. A., Bublitz, C. M., Hobgood, G. D., Martinez, M. N., Davis, J. L., Smith, G. W., et al. (2018). Effect of age on plasma protein binding of several veterinary drugs in dairy calves 2. Res. Vet. Sci. 121, 59-64. doi: 10.1016/j.rvsc.2018. 09.004

Nielsen, E. I., and Friberg, L. E. (2013). Pharmacokinetic-pharmacodynamic modeling of antibacterial drugs. Pharmacol. Rev. 65, 1053-1090. doi: 10.1124/ pr.111.005769

Nielsen, E. I., Viberg, A., Lowdin, E., Cars, O., Karlsson, M. O., and Sandstrom, M. (2007). Semimechanistic pharmacokinetic/pharmacodynamic model for assessment of activity of antibacterial agents from time-kill curve experiments. Antimicrob. Agents Chemother. 51, 128-136. doi: 10.1128/aac.00604-06

Sidhu, P., Rassouli, A., Illambas, J., Potter, T., Pelligand, L., Rycroft, A., et al. (2014). Pharmacokinetic-pharmacodynamic integration and modelling of florfenicol in calves. J. Vet. Pharmacol. Ther. 37, 231-242. doi: 10.1111/jvp.12093

Toutain, P.-L., Bousquet-Melou, A., Damborg, P., Ferran, A. A., Mevius, D., Pelligand, L., et al. (2017). En route towards european clinical breakpoints for veterinary antimicrobial susceptibility testing: a position paper explaining the VetCAST approach. Front. Microbiol. 8:2344. doi: 10.3389/fmicb.2017. 02344

Toutain, P.-L., Sidhu, P. K., Lees, P., Rassouli, A., and Pelligand, L. (2019). VetCAST method for determination of the pharmacokineticpharmacodynamic cut-off values of a long-acting formulation of florfenicol to support clinical breakpoints for florfenicol antimicrobial Susceptibility Testing in cattle. Front. Microbiol. doi: 10.3389/fmicb.2019.01310

Conflict of Interest Statement: The authors declare that the research was conducted in the absence of any commercial or financial relationships that could be construed as a potential conflict of interest.

Copyright (C) 2019 Pelligand, Lees, Sidhu and Toutain. This is an open-access article distributed under the terms of the Creative Commons Attribution License (CC BY). The use, distribution or reproduction in other forums is permitted, provided the original author(s) and the copyright owner(s) are credited and that the original publication in this journal is cited, in accordance with accepted academic practice. No use, distribution or reproduction is permitted which does not comply with these terms. 